\title{
A Review based on Brain Computer Interaction using EEG Headset for Physically Handicapped People
}

\author{
A F M Saifuddin Saif, MD. Ryhan Hossain, Redwan Ahmed, Tamanna Chowdhury \\ Department of Computer Science, Faculty of Science and Technology, American International University - \\ Bangladesh (AIUB)
}

Received: 21 October 2018; Accepted: 17 December 2018; Published: 08 March 2019

\begin{abstract}
The universes' most complex structure is the human brain. To analyze its characteristics, many studies and experiments have been carried out in a proper and systematic manner. From these researches and experiments, scientists have learnt to communicate with computer using brain and hence, BCI has been developed. A BrainComputer Interface (BCI) provides a communication path between the human brain and the computer system. With the advancement of information technology and neuroscience, there has been a flow of interest in turning fiction into reality. This research investigated existing works of BCI with the purpose developing a system that allows physically challenged people to communicate with other persons and helps to interact with the external environments with the help of computers. Components like, comparison of invasive and non-invasive technologies to measure brain activity, evaluation of control signals (i.e. patterns of brain activity or brain waves that can be used for communication), development or improvement of algorithms for translation of brain signals into computer commands, specific frequency components like electroencephalography (EEG), artificial neural network (ANN) etc. are used to accomplish such a feat. With such, the developments of new BCI applications are emerging every day.
\end{abstract}

Index Terms: Brain Computer Interface (BCI), Brain Wave, Electroencephalography (EEG), Artificial Neural Network (ANN).

(C) 2019 Published by MECS Publisher. Selection and/or peer review under responsibility of the Research Association of Modern Education and Computer Science.

\section{Introduction}

From the time when the computer was invented, people wanted to make it easier for everyone to control the computer. With that thought, people started to research and invented new technologies day by day just to make

* Corresponding author. A F M Saifuddin Saif

E-mail address: saif@aiub.edu 
the use of the computer easier than before. And the time came when technology evolved and scientists were able to develop a way using which physically handicapped people will be able to use computing devices. From that aspect Human Computer-Interaction (HCI) came followed by Brain-Computer Interface is introduced as improved phase.

With the advancement of technology, nowadays researchers have been able to make human brain interaction with computer more efficiently than before. Human brain emits electron signals called brain waves. Researchers have developed devices (EEG Headset) that can receive those brain waves and send it to the computer for further research. The brain wave signals are known as Electroencephalography (EEG) signals. The Electroencephalography (EEG) signals are generated in the brain through the voltage difference of ions moving through the neurons. There are thousands of neurons in a human brain. Each specific neuron generates some specific frequency for some specific command. The EEG signals are nothing but the brain activity in the form of electro-voltaic waves. Mostly the signals are used to monitor the brain activity of people. But nowadays EEG contains a fully developed sector which is known as BCI or Brain Computer Interaction. Through BCI, people can control computer and other devices just by thinking phases. In the previous researches, many researchers used EEG headset to collect the brain waves and then converted them to digital signals, some of them also used additional amplifiers that increase frequencies of the wave to get improved output. Then the signals went through an AI system which converts them into executable commands.

Currently, electrophysiological signals used in BCI can be described into three categories: specific frequency components of EEG produced spontaneously during mechanism of brain thinking, such as alpha waves, beta waves and so on; second category is brain evoked Event- Related Potentials (ERP), i.e. neural electrical activity of cerebral cortex induced by specific sensory stimulus or event operation and third category is the electrical activity signals of neurons in group directly acquired from electrode implanted into the specific region of the brain cortex. Using these waves BCI applications have been made possible. EEG signals have mostly four wave patterns, i.e. Alpha, Beta, Theta and Delta. Alpha waves are produced during a person is thinking about anything consciously. Beta waves are generated when a person feels anxious, worried or angry, mostly when the user's state of mind changes and Delta waves are generated subconsciously by a person. And finally, theta waves and some delta waves are generated when user is having a dream. During the recording of the signals, sometimes Amplitude of the signal reaches an observable length. The observable peak is called Event Related Potential (ERP). ERP defines that an event has occurred in the brain or a thought has been generated in the brain. This research illustrates investigation that has been concluded on Brain Computer Interaction.

\section{Related Work}

With the help of BCI, the use of computer is becoming easier even for people with physical disabilities. More and more researches are done based on BCI. Researchers have already developed systems that will help the disabled people to control computers like home appliances, wheelchair, mobile phone, and more developed systems are coming every day.

There are many ways using which researchers are developing BCI technology but the main equipment for a BCI device is an EEG headset which is used by many researchers on many researches. The process is explained in steps; at first, brain signals needs to be collected for processing purpose. Signal acquisition involves collecting the electroencephalography signals generated in the human brain. EEG signals are generated due to the electrical activity on the brain scalp. Under the scalp, there is a flow of ionic current which are collected through the EEG head set as brain signals. There are two ways to collect the brain waves first one is inverse and the second one is non-inverse. In the inverse method, the electrodes need to put inside one's brain to collect the brain waves. It is more accurate and gives some clear signals without any noise but it is more dangerous for the users. For the second method, the user only needs to put the headset on his/her head to get the readings. This method is not as accurate as the previous one but it is safer. For many researches, the second method has been chosen.

Up until now, researchers have developed a number of systems to control computing activity using a number 
of ways. Shanmugapriya B. et al., 2014, Hundia ,2015, G. Ambica and B. Sujatha, 2015, U. Hoffmann et al.,2008 have developed systems that allow users both able-bodied and physically challenged to control computer using EEG technology [1, 2, 4, 15]. Ramesh C R and Prof. Lyla B Das, 2015 have developed an interface that will help a speech impediment person to speak using BCI device [10]. Sneha Pushpa $S$ and Chandrashekar N S ,2007, B. SUJATHA and G. AMBICA ,2015, Nikhil R. Folane and R. M. Autee, 2016, P. kumar et al., 2017 have developed interface using which home appliances can be controlled [6,8,12,18]. V. Rajesh Kannan and K. O. Joseph, 2004, Jaromir Svejda et al., 2015, Kale Swapnil T. et al., 2014, T. Prasanth et al., 2017 have developed robot controlling system using BCI technology [7, 13, 17, 20, 25]. Pradeep Kumar et al have developed an interface that allows the user to control smart phone [11]. Using P300 Event-Related Potential Kale Swapnil T. et al., 2014 have developed a system through which people will be able to control internet browser [16]. R. Chandana Priya and K. Aparna, 2017, S. Naresh Babu and G. Nagarjuna Reddy, 2015 have developed mind-controlled wheelchair for disabled people using BCI [21, 24]. Even computer gaming has been done using BCI technology. Srinath Vikramakumar and Dr. V. Dhanakoti, 2017 have developed a system that allows users to play a computer game using their mind [22]. More and more technologies are being developed with modern technology. This research intends to investigate previous methods, frameworks that previous researchers have used and the results they achieved for validation.

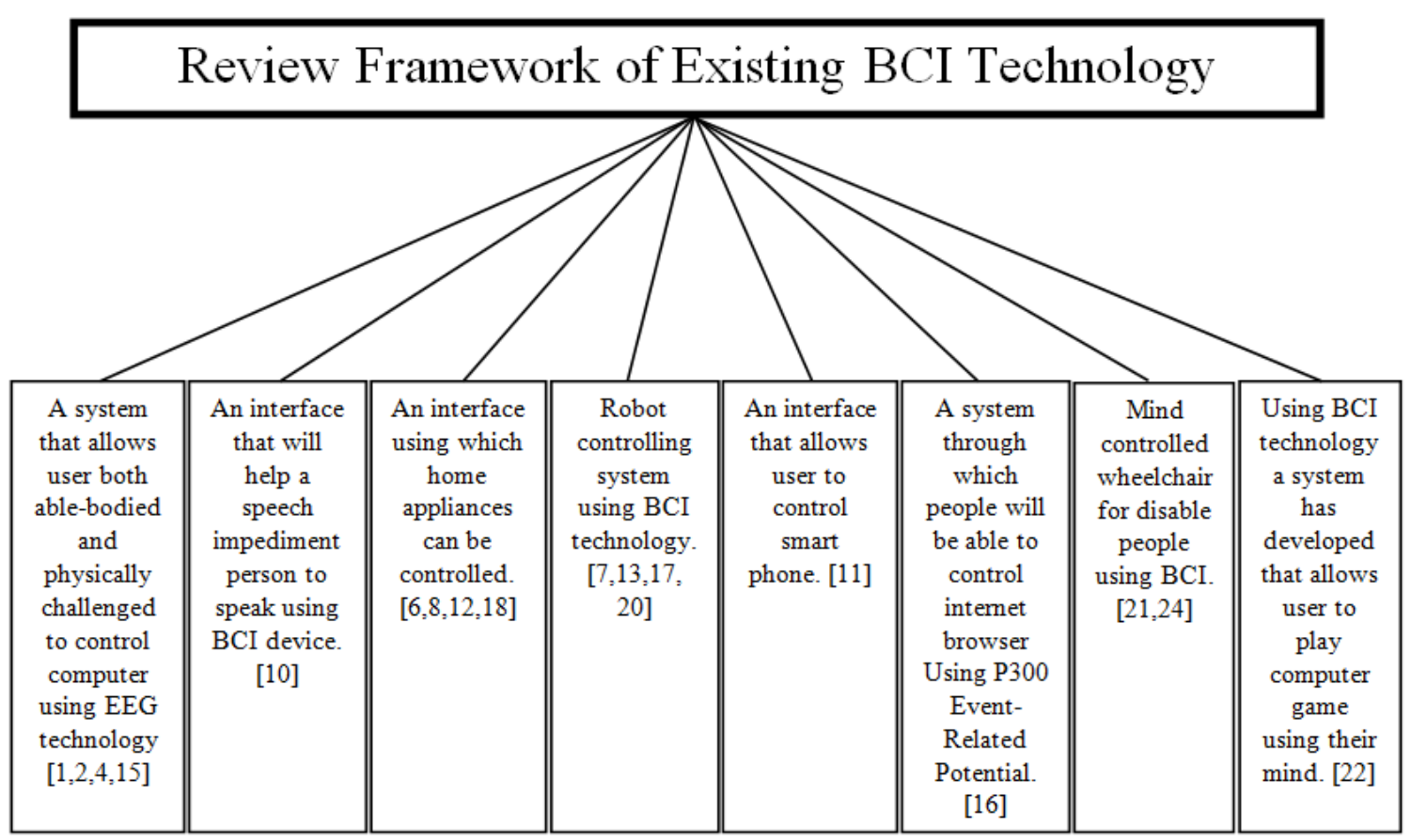

\section{Review based on Methods}

Most of previous research used Electroencephalography (EEG) based biomedical signal processing system to perform computer operations by manipulating the brain activity. The users have to explicitly manipulate the brain activity to produce signals that can be used to operate the computer. The brain waves are obtained with the help of scalp electrodes. EEG signals are collected and then processed to interpret the command and execute the desired task.

To control computer with thinking is not a fiction. It is now possible by using BCI and researchers have 
proved that computer can be controlled using mind, let it be controlling a wheelchair, home appliances, personal computer or even playing a game. Table 1 represents different methods used to make fiction into reality.

Table 1. Qualitative Study of Different Methods based on Brain Computer Interaction.

\begin{tabular}{ccc}
\hline Method & Advantages & Disadvantages \\
\hline $\begin{array}{c}\text { Artificial Neural Network (ANN) } \\
{[1,12,20]}\end{array}$ & $\begin{array}{c}\text { Reduces the fatigue of normal computer } \\
\text { users. }\end{array}$ & $\begin{array}{c}\text { The system must undergo training to } \\
\text { understand user's thoughts. }\end{array}$ \\
\hline Fast Fourier Transformation (FFT) $[2,4]$ & $\begin{array}{c}\text { The system can be used by people to gain } \\
\text { thought process control over various } \\
\text { robotic systems. }\end{array}$ & $\begin{array}{c}\text { The system doesn't chiefly work on the } \\
\text { basis of measuring the FFT and doesn't } \\
\text { measure the amplitude and the frequency } \\
\text { response curve of the brain signals. }\end{array}$ \\
\hline Event-Related Potential (ERP) [10] & $\begin{array}{c}\text { ERP provides measures of neural activity } \\
\text { with very high temporal resolution. }\end{array}$ & $\begin{array}{c}\text { Only sufficiently strong potentials } \\
\text { generated synchronically by open fields } \\
\text { are recordable across the scalp }\end{array}$ \\
\hline Non-invasive BCI $[3,6,8,13,17,19]$ & Very fast & Least signal clarity \\
\hline P-300 $[15,16]$ & Requires no user training & $\begin{array}{c}\text { The P300 can be influenced by different } \\
\text { human factors such as attention, } \\
\text { motivation and fatigue. }\end{array}$ \\
\hline
\end{tabular}

With EEG signals and different methods, researchers have developed systems that can help physically challenged people in a very adaptable manner. Using artificial neural network, a computer controlling system has been developed. However, for the neural network to work properly, the system is needed to go through training. If the training set is not good enough, the result may also not be good. While training is a problem, P300 can be used which requires no training for the users who would use the devices. Using FFT, previous researchers developed a system that can help physically disabled people to control robotic movements like prosthetic arm and leg, wheelchair, and even some movements of a multipurpose robot. Even though FFT is used in variety of researches, its response is slow. However, non-invasive BCI can be used here as it is very fast but it has the least signal clarity. Event-related potential provides measures of neural activity with very high temporal resolution but one of the main limitations of the ERP technique is that only sufficiently strong potentials generated synchronically by open fields are recordable across the scalp.

\section{Review based on Frameworks}

When it comes to Brain Computer Interaction, brainwave is the power source of all research. In most of the research framework, brainwave is collected from the user and after implementing different algorithms, the data is sent to the computer or any other devices that are wished to be controlled. Shanmugapriya et al., 2014 collected brainwave of users and performed computer operations using neural network [1]. Shanmugapriya et al.(2014), Nikhil R. Folane and R. M. Autee, Madhu Nakirekanti et al., 2016 also developed their system using neural network $[12,20]$. But for their system to give the best result, training data set for the system has to be rich, otherwise result will not be satisfactory. Hundia, 2015 did the same thing but he specifically used alpha brainwave to accomplish his task [2]. Ramesh C R and Prof. Lyla B Das, 2015 used brainwave to accomplish voice for speech impediments by sending the wave to a signal processing unit [10].

The brainwave signal has also been used to remotely control devices as well. Sneha Pushpa $\mathrm{S}$ and Chandrashekar N S , 2007 used brainwave to control home appliances using Bluetooth system by transmitting the wave to an ARM 7 processor [6]. V. Rajesh Kannan and K. O. Joseph, 2004 have also used the Bluetooth technology to control mobile robot using brainwave [7]. But the response of the device is slow. To solve that problem Kale Swapnil T et al., 2014 used non-invasive BCI technology which is very fast [17]. They extracted the features of the neural signal collected from the user, decoded the signals and then send it to a navigation system to control robot navigation using non-invasive BCI.

Just like Kale Swapnil T et al., 2014, Mr. Ramesh C R and Prof. Lyla B Das, 2015, Sneha Pushpa S and 
Chandrashekar N S, 2007, G. Ambica and B. Sujatha, 2015, Jaromir Svejda et al., 2015, Wei Tuck Lee et al., 2013 have also used non-invasive BCI to get fast response [3, 6, 8, 13, 19]. Even though non-invasive BCI is fast, using it gives the least signal clarity. This problem can be solved using ERP as it provides measures of neural activity with very high temporal resolution.

Pradeep Kumar et al., 2017 used the brainwave to make an assistive framework using a smartphone by using neurosky headset and sending the brainwave data to the phone using Bluetooth [11]. Nikhil R. Folane and R. M. Autee, 2016 made a brainwave controlled wheelchair control system by sending the brainwave to a computer system where the brainwave is analyzed using MATLAB, level analyzer and finally using decision maker, the data is sent to the wheelchair through ARM processor [12]. Jaromir Svejda et al., 2015 also made a robot movement controlling system using brainwave by using neuro headset, translation algorithm and sending the data using Bluetooth device [13]. But as they used non-invasive BCI, the response is fast in comparison with other frameworks.

Emily Mugler et al., 2008 used brainwave to control internet browser of a computer by amplifying the brainwave collected from users and using their add-ons for Mozilla Firefox internet browser using P300 EventRelated Potential [16]. Usage of P300 gave them an extra advantage as they did not need to train the users to use the system. But P300 can be influenced by different human factors such as attention, motivation and fatigue. Hence, it might not give the best response all the time.

\section{Review based on Experimental Results}

Akshaya T. et al., 2014 proposed work is an Electroencephalography (EEG) based biomedical signal processing system to perform computer operations by manipulating the brain activity [1]. The users have to explicitly manipulate brain activity to produce signals that can be used to operate computer. The brain waves are obtained with the help of scalp electrodes. EEG signals are collected and then processed to interpret the command and execute the desired task. The real-time implementation requires training in the computer according to one's thoughts and actions through a neural network. The main advantage of their work is that it reduces operation difficulty. Hundia (2015) developed a system that can be used by the differently abled people to gain thought processed control over various prosthetics, wheelchairs and functioning robotics systems [2]. But the main weakness of their research was the lack of accurate data to compare the accuracy of their system. Mr. Ramesh C R and Prof. Lyla B Das, 2015 achieved 81.17\% accuracy and also when the device is active and taking command, it goes to attention mode and for idle time, it goes for mediation mode. Also Computing et al., 2015 went through a proper FFT algorithm analysis [4] that was not mentioned in [1, 2, 3].

The program described by J. Katona et al., 2014, enable users to investigate how brain wave signals are measured by the EEG headset - alternate in time and how they depend on the changes of brain activity [5]. On the basis of the results, the information obtained by the processed brain waves can be used in several research areas, for instance, medical research, multimedia applications, games etc. But when it comes to using only home appliance Sneha Pushpa S and Chandrashekar N S, 2007 presented the design and implementation of non-invasive type of Brain-Computer Interface technique to control home appliances using EEG based brain signals that works on controlling the physical devices, so the paralyzed and the physically disabled people can independently do their work like switching on and off the lights and fans by their own [6]. This result mostly depends on one person's attention level which cannot be the same all the time. G. Ambica and B. Sujatha ,2015 used eSense Attention meter and showed intensity of a user's level of mental "focus" or "attention", such as that which occurs during intense concentration and directed (but stable) mental activity. Its value ranges from 0 to 100 [8]. Distractions, wandering thoughts, lack of focus, or anxiety may lower the attention meter level. With trained system, accuracy can be increased significantly. Ramesh C R and Prof. Lyla B Das, 2015 experimented the results by showing that even without training, the average accuracy obtained is around $67 \%$ [10]. Their research expected that if proper training is done before testing, the accuracy can be increased to $100 \%$ which shows that the idea implemented can be very useful in real life for the intended people.

The use of regular algorithm DFT which Pradeep Kumar et al., 2017 showed in DFT analysis of the captured 
signals has been performed to extract useful features [11]. Recognition of 9 mental commands from 8 participants has been performed using HMM classifier with an accuracy of $68.69 \%$ but in the other hand using the alpha wave to recognize eye blinking Jaromir Svejda et al., 2015 found that the average reliability of robotic device response on the signal of eye blinking was $65.45 \%$. On the other hand, the response on the state of open eyes was around $98 \%$ but Nikhil R. Folane and R. M. Autee, 2016 found out that with $70 \%$ concentration user can control a wheelchair [12]. But the response of the wheelchair is slower than expected which is less accurate. In case of disabled subjects, Ulrich Hoffmann et. (2008) was able to classify $100 \%$ accuracy which was done for their subjects [15]. But in such case, they clearly showed an acceptable result but some of the previous research resultant were not able to provide any perimeter data they had used and also there was no evidence of how they used it while only the possible outcome had shown $[16,17,18,19]$.

For using the EEG headset for normal people, R. Chandana Priya, K. Aparna, 2017 used eye blinking giving a close result of command execution. Besides, the proposed system demonstrated the possibility of forecasting the disabled people's vigilance in real time. The proposed work uses a hearing assistance from a single electrode that makes the wheelchair to perform four operations (right, left, and front, back) without GUI unless system requires any control device with high-level power [21]. But T. Prasanth et al., 2017 device allowed paralyzed people to move around by using their mind. It transmits the auditory data to the mind of a deaf person and allows them to hear. By using our mind, we may control video games also [25]. Both of their devices have some issues, such as there is an occurrence of harmful effects on the environment by using these technologies. They get very few electric signals by placing electrodes in Non - invasive manner. By placing electrodes in an invasive manner creates a scar tissue in the brain.

In case of BCI based gamming Srinath Vikramakumar and Dr. V. Dhanakoti's, 2017 described the application of brain wave signal in gaming controller [22]. The controller system was completely hands-free and made it usable for people with disability but the description only showed how the brain reacts when the game starts. Table 2 shows performance summarization for different datasets.

Table 2. Performance Summarization of Different Methods for Different Datasets

\begin{tabular}{|c|c|c|}
\hline Method & Dataset & Accuracy/Reliability \\
\hline $\begin{array}{c}\text { Fast Fourier Transform (FFT), } \\
\text { Neural Network[1] }\end{array}$ & $\begin{array}{l}\text { Electrooculography (EOG) } \\
\text { Electromyography (EMG) }\end{array}$ & Triticale explanation \\
\hline Fast Fourier Transform (FFT)[2] & Not Available & Response delay was 2.89 seconds \\
\hline Event-Related Potential ERP[3] & $\begin{array}{c}\text { Datasheet of PCF8574AN } \\
\text { Datasheet of L293D } \\
\text { Datasheet of ISD 25120 } \\
\text { Addressing modes of ISD 25120 } \\
\text { Datasheet of LM386 }\end{array}$ & Average accuracy $81.17 \%$ \\
\hline Event-Related Potential ERP[10] & $\begin{array}{c}\text { Datasheet of PCF8574AN } \\
\text { Datasheet of L293D } \\
\text { Datasheet of ISD 25120 } \\
\text { Addressing modes of ISD 25120 } \\
\text { Datasheet of LM386 }\end{array}$ & Average accuracy $67 \%$ \\
\hline eSense[8] & Data Level analyzer unit (LAU) & $80 \%$ accuracy \\
\hline Emotiv SDK[13] & Not Available & $98 \%$ when the user's eye is open \\
\hline $\begin{array}{l}\text { Discrete Fourier Transform } \\
\text { (DFT)[11] }\end{array}$ & Hidden Markov Model (HMM) & accuracy of $68.69 \%$ \\
\hline $\begin{array}{l}\text { Artificial Neural Network } \\
\text { (ANN)[12] }\end{array}$ & Not Available & $70 \%$ average concentration \\
\hline $\mathrm{P} 300[15,16]$ & http://bci.epfl.ch/p300 & $100 \%$ accuracy \\
\hline
\end{tabular}

\section{Observation and Discussion}

For BCI, most researchers used EEG headset. The gathered brainwave is then run through different 
algorithms and transformed to computer language to control computing activity. When it comes to a single method or framework, all of them have advantages and disadvantages. When ANN can be used to make a system easier to use for users, it also needs to train the system using the best possible dataset. When NonInvasive BCI is used for faster service, it has the drawback of not being able to get clear signal. ERP, with the ability to get better signal data, it has the drawback of having the ability to get the data only across the scalp.

When it comes to the results, systems developed using FFT are suited for physically disabled persons but lacks the communication speed [2]. Using ERP, the accuracy rate can be increased but it would need the users to be trained $[3,10]$. Even though ANN is better for users, researchers have found only $70 \%$ accuracy when users are concentrating [12]. Such results found from previous research that a system using Emotiv SDK gave 98\% accuracy but the user has to keep their eye open. Up until now, best result has been shown by the system using P300 based BCI and accuracy rate is $100 \%$.

Above all most of the previous research did not show the parameter and also any numerical values because EEG headset basically does that task for them. Furthermore, almost all of them used either DFT or the IDEFT algorithm for detecting brain waves and converting them. And as for noise reduction, backpropagation or other algorithms can be used. Adding an amplifier can also prevent the relic frequencies from interfering. NeuroSky EEG headset, mind wave headset by Neurosky and NeuroSky Brainwave sensor are basically the same but different version of the EEG headset used in previous research. Also, the main difference exists among research is mostly the different placement and number of scalps and the size. Many of the projects are based on only the alpha wave but for several command executions there is also need for Beta waves which was negated in many cases.

\section{Conclusion}

Above all, previous research contains one specific sequence regarding way of performing various tasks related to brainwave. Almost all of the researchers have used Electroencephalography (EEG) headset to collect brainwave data. The EEG headset collects brain signals and then converts them to digital signals. The researchers may decide which type of brainwave they may accept (i.e. Alpha, Beta, Delta, Theta) and which may discard and then it will be sent to a computer to process the signal with reference to the analysis of the data. The subjects are quite possible to use the alpha or several types of brainwaves to gain control over electronic devices. By recording the data over a few seconds, the subject can gain control over devices like robots, wheelchair and other smaller devices. With this system, the user does not have to use much body muscle movements. Hence, these types of systems or interface are best suited for physically disabled people. So this research finally remarks based on previous research investigation that there is room for further improvement in the BCI sector. The negligence of Beta waves, the command execution and the response time needs to improved further more. The noise margin is also needed to be improved. With more research and advanced investigation, physically disabled people who cannot even walk but only has brain activity may one day be able to communicate with the world like any other normal person with the help of Brain-Computer Interface.

\section{Acknowledgements}

This research immensely supported through knowledge sharing from ABERT Research Team (AIUB Brain Engineering Research Team) in the Department of Computer Science, American International University Bangladesh (AIUB). 


\section{References}

[1] S. B, Akshaya T, K. K, and Anbarasu V, "Controlling Computer Operations using Brain-Wave Computing”, International Journal of Computational Engineering Research, vol. 4, pp. 76-81, 2014.

[2] R. Hundia, "Brain Computer Interface-Controlling Devices Utilizing The Alpha Brain Waves", International Journal of Scientific \& Technology Research, vol. 4, no. 1, pp. 281-285, 2015.

[3] Ramesh C R, L. B. Das, "Brain Computer Interface Based Assistive Device", 2015 Int. Conf. Adv. Comput. Commun. Informatics, pp. 330-334, 2015.

[4] M. Computing, G. Ambica, and B. Sujatha, "STUDY AND APPLICATION OF BRAIN WAVES (ALPHA, BETA ) FOR USER AMBIENT ENVIRONMENT CONTROL”, vol. 4, no. 10, pp. 197-200, 2015.

[5] J. Katona, I. Farkas, T. Ujbanyi, P. Dukan, and A. Kovari, "Evaluation of the NeuroSky MindFlex EEG headset brain waves data", SAMI 2014 - IEEE 12th Int. Symp. Appl. Mach. Intell. Informatics, Proc., pp. 91-94, 2014.

[6] S. Pushpa, C. N. S, and P. G. Student, "EEG based Brain-Computer Interface for Controlling Home Appliances”, Int. J. Innov. Res. Sci. Eng. Technol. (An ISO Certif. Organ., vol. 3297, no. 6, pp. 98709877, 2007.

[7] V. Rajesh and K. O. Joseph, "Brain Controlled Mobile Robot Using Brain Wave Sensor", pp. 7782,2004 .

[8] B. Sujatha and G. Ambica, "EEG Based Brain Computer Interface for Controlling Home Appliances", Int. Res. J. Eng. Technol., pp. 2395-56, 2015.

[9] S. G. Mason and G. E. Birch, "A general framework for brain-computer interface design", IEEE Trans. Neural Syst. Rehabil. Eng., vol. 11, no. 1, pp. 70-85, 2003.

[10] Mr. Ramesh C R, Prof. L. B. Das, "Brain Computer Interface Device for Speech Impediments", 19-21 November 2015, Int. Conf. on Controll, Communication \& Computing India (ICCC).

[11] P. Kumar, R. Saini, P. K. Sahu, P. P. Roy, D. P. Dogra, and R. Balasubramanian, "Neuro-phone: An assistive framework to operate Smartphone using EEG signals", 2017.

[12] N. R. Folane, R. M. Autee "EEG Based Brain Controlled Wheelchair for Physically Challenged People", International Journal of Innovative Research in Computer and Communication Engineering, Vol. 4, Issue 1, January 2016.

[13] J. Svejda, R. Zak, R. Senkerik, and R. Jasek, "Using Brain - Computer Interface for Control Robot Movement", vol. 8, no. Cd, 2015.

[14] A. L. S. Ferreira, L. C. de Miranda, E. E. C. de Miranda, and S. G. Sakamoto, "A Survey of Interactive Systems based on Brain-Computer Interfaces”, SBC J. Interact. Syst., vol. 4, no. 1, pp. 3-13, 2013.

[15] U. Hoffmann, J. M. Vesin, T. Ebrahimi, and K. Diserens, "An efficient P300-based brain-computer interface for disabled subjects", J. Neurosci. Methods, vol. 167, no. 1, pp. 115-125, 2008.

[16] Emily Mugler, Michael Bensch, Sebastian Halder, Wolfgang Rosenstiel, Martin Bogdan, Niels Birbaumer, Andrea Kübler, "Control of an Internet Browser Using the P300 Event-Related Potential", International Journal of Bioelectromagnetism, Vol. 10, No. 1, pp. 56 - 63, 2008.

[17] Kale Swapnil T, Mahajan Sadanand P, Rakshe Balu G, Prof. N.K.Bhandari, "Robot Navigation control through EEG Based Signals", International Journal Of Engineering And Computer Science, ISSN:23197242, Volume 3, Issue 3, Page No. 5109-5113, March-2014.

[18] Praveen kumar, M.Govindu, A.Rajaiah, "Automatic Home Control System Using Brain Wave Signal Detection”, IJESC, ISSN-2321-3361, 2014.

[19] Wei Tuck Lee, Humaira Nisar, Aamir S. Malik, Kim Ho Yeap, “A Brain Computer Interface for Smart Home Control”, 2013 IEEE 17th International Symposium on Consumer Electronics (ISCE). 
[20] Madhu Nakirekanti, Dr. Raja Murali Prasad, Eliyaz Mahammad, K. Narsimha Reddy, "BRAIN WAVE CONTROLLED ROBOT USING MATLAB", International Journal of Mechanical Engineering and Technology (IJMET), Volume 8, Issue 12, pp. 750-759, Article ID: IJMET_08_12_081, December 2017.

[21] R. ChandanaPriya, K. Aparna, "MIND WAVE SENSOR CONTROLLED WHEEL CHAIR", International Journal of Advance Engineering and Research Development, Volume 4, Issue 9, September -2017.

[22] Srinath Vikramakumar and Dr. V. Dhanakoti, "Mind wave Based Gaming Controller Using User Friendly EEG Signal Acquisition", International Journal of Innovations \& Advancement in Computer Science, ISSN 2347 - 8616, Volume 6, Issue 11, November 2017.

[23] Dipti Pawar, Kapil Kamale, Ishaan Singh, Vishwajeet Kharote, Prajwal Goswami., “A Survey on Thoughts to Text Using Brainwave Patterns", International Journal of Innovative Research in Computer And Communication Engineering, Vol. 5, Issue 5, May 2017.

[24] S. Naresh Babu, G. Nagarjuna Reddy, "AN INTELLIGENT ROBOT CONTROL USING EEG TECHNOLOGY", International Journal of Application or Innovation in Engineering \& Management (IJAIEM), Volume 4, Issue 3, March 2015.

[25] T. Prasanth, P. Dharma Teja, K. DasmanthaRao, M. N. Shyam Kumar, M. Rama Krishna, "CONTROLLING OF ROBOT USING BRAIN SIGNALS WITH MATLAB", SSRG International Journal of Computer Trends and Technology (IJCTT), ISSN: 2231-2803, Special Issue, April 2017.

[26] B. Sivakumar and K. Srilatha, "A novel method to segment blood vessels and optic disc in the fundus retinal images”, Res. J. Pharm. Biol. Chem. Sci., vol. 7, no. 3, pp. 365-373, 2016.

\section{Authors' Profiles}

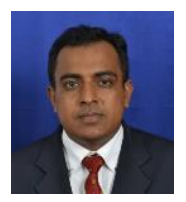

A.F.M. Saifuddin Saif received PhD from Faculty of Information Science and Technology, University Kebangsaan Malaysia (UKM) in 2016. He received M.Sc. in Computer System Engineering (Software System) from University of East London, UK and B.Sc. (Eng.) degree in Computer Science and Engineering from Shahjalal University of Science and Technology, Bangladesh in 2012 and 2008, respectively. Most of his contributions in Computer Vision and Artificial Intelligence Research field were published in ISI Q1 journals. He has published many papers in ISI indexed Journals, Scopus indexed Journals, Book Chapters, Conferences and Proceedings. He served as Technical Committee Members, Reviewers, Guest Speakers, Session Chairs in many Conferences and Workshops. Currently, Dr. A.F.M. Saifuddin Saif is an Assistant Professor at Faculty of Science and Technology, American International University - Bangladesh. Before joining the university, he did Post Doctorate at Faculty of Information Science \& Technology, University Kebangsaan Malaysia. He spent more than 6 years in IT industry such as Advanced Software Development, Web eMaze etc as IT researcher. His research interests include Image Processing, Computer Vision, Artificial Intelligence, Augmented Reality, 3D reconstruction and Medical Image Processing.

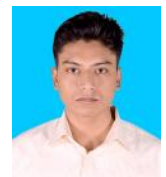

MD. Ryhan Hossain is an undergraduate student of Computer Science and Engineering (CSE) program at the Faculty of Science and Information Technology of American International University - Bangladesh (AIUB). He is currently also serving as Teaching Assistant in the Department of Computer Science. His research interests and passion are mostly based on Brain Computer Interaction, Artificial Neural Network, Computer Vision, Machine Learning, Data

mining. 
Redwan Ahmed Rahul is an undergraduate student of Computer Science and Engineering (CSE) program at the Faculty of Science and Information Technology of American International University-Bangladesh (AIUB). His research interests and passion are mostly based on Convolutional Artificial Neural Networks, Brain Computer Interface, EEG signal processing also included in the field of Computer Vision, Image Processing and Machine Learning.

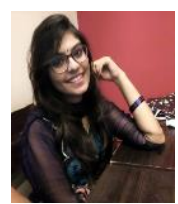

Tamanna Chowdhury is an undergraduate student of Computer Science and Engineering (CSE) program at the Faculty of Science and Information Technology of American International University - Bangladesh (AIUB). She has received Dean's List Honors Award 2017 \& 2016 for her academic performance. Her research interests and passions are mostly based on Brain Computer Interaction, Artificial Neural Network, Machine Learning, Data mining.

How to cite this paper: A F M Saifuddin Saif, MD. Ryhan Hossain, Redwan Ahmed, Tamanna Chowdhury,"A Review based on Brain Computer Interaction using EEG Headset for Physically Handicapped People", International Journal of Education and Management Engineering(IJEME), Vol.9, No.2, pp.34-43, 2019.DOI: 10.5815/ijeme.2019.02.04 